\title{
Short term response of patients with bronchiectasis to treatment with amoxycillin given in standard or high doses orally or by inhalation
}

\author{
SL HILL, HM MORRISON, D BURNETT, RA STOCKLEY \\ From the Lung Immunobiochemical Research Group, General Hospital, Birmingham
}

ABSTRACT The effect of three amoxycillin treatment regimens on purulent secretions of patients with bronchiectasis has been studied. On the basis of information recorded on a diary card the patients were divided into three groups, according to the usual nature of their secretions: seven who produced mucoid sputum, which occasionally became purulent; seven whose secretions were usually mucopurulent but occasionally purulent; and 19 whose secretions were persistently purulent. Treatment with capsules of amoxycillin in a dosage of $250 \mathrm{mg}$ three times a day resulted in clearance of purulent secretions in patients of the mucoid group when they were treated for a clinical exacerbation. The sputum remained clear in these patients for long periods before a further exacerbation (median 61/2, range 1-11 months). The mucopurulent-purulent group also responded to this dosage but sputum purulence returned more rapidly (median 9, range 4-31 days). Only three of the $19(17 \%)$ patients with persistently purulent secretions showed a macroscopic response to this dosage, whereas seven $(60 \%)$ of 12 patients who received the higher dosage ( $3 \mathrm{~g}$ sachets twice a day) responded. Among the failures, some responded to nebulised amoxycillin, suggesting that higher levels of amoxycillin in secretions are required in these patients. Macroscopic clearance of purulent secretions was finally achieved in most of the patients studied. The response was not always predictable from the results of sputum culture. Clearance of secretions by antibiotics was also identified by the patients, using a diary card score. Improvements in well being and in symptoms were noticed even in the group who usually produced mucopurulent and purulent secretions even though they appeared to be clinically stable before treatment.

The role of antibiotic treatment in the management of bronchiectasis is uncertain and, despite the report of the Medical Research Council working party ${ }^{1}$ suggesting that continuous treatment was beneficial, these patients are usually treated only when they have exacerbations associated with increased symptoms, pyrexia, and chest pain. We have recently provided evidence, however, that the purulent secretions of such patients may be harmful to the lung and be associated with worse lung function. ${ }^{2}$ Furthermore, the secretions have been shown to have a deleterious effect on cilia in vitro ${ }^{3}$ and could be responsible for the decreased mucocilliary clearance seen in these patients. ${ }^{4}$ Antibiotic treatment was shown to reverse

Address for reprint requests: Dr RA Stockley, General Hospital, Birmingham B46NH.

Accepted 10 December 1985 the potentially harmful nature of these secretions ${ }^{35}$ even when given in the apparently stable clinical state.

Not all of the patients studied previously ${ }^{5}$ responded to their initial course of antibiotics and it has been suggested that patients with persistent bronchial sepsis may require higher doses of antibiotic than those conventionally prescribed. ${ }^{6}$

The present study was designed to investigate several aspects of the response to antibiotic treatment in a group of patients with bronchiectasis. In particular, we wished to determine what dosage of antibiotic was necessary to clear the secretions and whether the dose requirement could be predicted. We also wished to assess the patients' clinical response and the rapidity with which they returned to the pretreatment state. In this way we hoped to gain information that would help to determine whether the use of larger doses of antibiotic might be appropriate in some patients with bronchiectasis. 


\section{Methods}

We studied 33 patients ( 21 female) aged $29-76$ years with a clinical diagnosis of bronchiectasis. Eighteen patients had bronchograms and the remaining $15 \mathrm{had}$ radiological evidence of bronchial wall thickening with cystic changes. Twenty five were lifelong nonsmokers and seven were ex-smokers, having stopped smoking at least two years previously. Seven patients had previously undergone lobectomy for the disease. Two patients had panhypogammaglobulinaemia.

The mean $\mathrm{FEV}_{1}$ was 1.541 (SD 0.79) and the mean $\mathrm{FEV}_{1}$ as a percentage of the forced expiratory vital capacity (FVC) was $53.1 \%(15.76 \%)$. Only two patients showed reversibility of a $\beta_{2}$ agonist (four puffs by metered dose inhaler) (increase in $\mathrm{FEV}_{1} 15 \%$ and $21 \%$ ).

Throughout the study all patients continued their usual postural drainage routine and other prescribed treatment. Patients gave informed consent to the studies, which were approved by the hospital ethical committee. The study was divided into two partsassessment of the pattern of disease and the response to antibiotic treatment.

\section{ASSESSMENT OF THE PATTERN OF DISEASE}

All 33 patients completed a daily diary card to monitor the nature of secretions, symptoms, and peak expiratory flow (PEF) (best of three attempts on rising, with a Wright's mini peak flow meter). The diary card recorded the following:

\section{Sputum characteristics}

Colour, volume, and thickness were noted on a point scale. The scales were:

Colour 1-mucoid (colourless); 2-pale yellow or pale green; 3-dark yellow or dark green.

Volume 0 -none; 1 -up to one eggcupful a day; 2-more than one eggcupful to half a cupful a day; 3 -more than half to one cupful a day.

Thickness 1-watery; 2-sticky liquid; 3-semisolid.

\section{General symptoms}

Chest pain was noted as being present or absent. Cough, breathing, and well being were noted on a point scale:

Cough 1 = occasionally, 2 = persistently. Breathing and well being 1-good; 2-slightly bad; 3-bad.

The diary cards were initially kept for at least two weeks, during which they were checked for consistency by one of us (SLH) and any problems of interpretation were identified and corrections made.

From this early part of the study the 33 patients could be classified into three groups according to the usual nature of secretions produced when they were apparently clinically well: (1) those who produce persistently purulent sputum every day (PP) -19 永 patients; (2) those who usually produce mucoid spu- 7 tum (M)-seven patients; (3) those whose sputum usually varies between purulent and mucopurulent (MP/P) - seven patients.

\section{ANTIBIOTIC TREATMENT}

After the initial classification of sputum we assessed the response of the 33 patients to antibiotic treatment.

Antibiotics were prescribed for the following indications: (1) clear exacerbations - sputum becoming purulent, increased breathlessness and cough, and general malaise in patients usually producing mucoid sputum; (2) clinically stable patients producing purulent sputum either persistently (PP) or intermittently (MP/P).

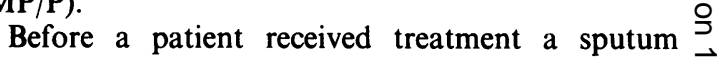
sample was collected during the first four hours after $\vec{c}$ waking. This was sent for routine bacteriological culture after macroscopic assessment of purulence by $\vec{\bullet}$ one of us (SLH). This procedure was repeated both during and after antibiotic treatment for all treatment regimens.

A response to treatment was taken as a clear change in sputum colour from purulent to mucoid or slightly mucopurulent as assessed by SLH. The time to relapse was assessed as the time taken for the sputum purulence to return. This was confirmed from a weekly sputum assessment by SLH.

Initially all 33 patients, none of whom had a known history of penicillin sensitivity, received low dose amoxycillin $(250 \mathrm{mg}$ capsules three times a day for 14 days). At the end of the treatment they were judged as having either responded or not responded on the basis of the assessment by SLH. If a response had occurred the time to relapse was assessed.

Patients who failed to respond (sputum remaining purulent) were left for a period of two weeks. They then received high dose amoxycillin ( $3 \mathrm{~g}$ sachets dissolved in water, twice a day for 14 days). At the end of treatment they were again judged as having responded (in which case the time to relapse was assessed) or not responded. Any patient failing to respond to this regimen was reviewed several months later and received nebulised amoxycillin (as described previously ${ }^{7}$ ). After four months of treatment their response and subsequent relapse was assessed.

The percentages of patients responding (on the basis of the macroscopic assessment by SLH) to the ? three amoxycillin regimens were obtained for the 0 whole group and each of the three patient subgroups described earlier (M, MP/P, and PP).

DIARY CARD

Twenty nine of the patients $(6 \mathrm{M}, 7 \mathrm{MP} / \mathrm{P}, 16 \mathrm{PP})$ had 
continued to complete daily diary cards and these were used to determine their assessment of the effect of antibiotic treatment. The patients were divided into responding and non-responding groups (both oral regimens) on the basis of the macroscopic assessment by SLH. The diary cards were analysed independently in retrospect. The mean of three consecutive days' scores for well being; breathing; cough; chest pain; and sputum colour, volume, and thickness were obtained immediately before treatment, during the last three days of treatment, and two weeks after treatment had stopped. For the purpose of statistical analysis, chest pain was given a numerical score-1 for present and 0 for absent. Frequency tables were constructed for each symptom score and differences between two time points were determined by $\chi^{2}$ analysis.

The average peak expiratory flow was also noted for the same three days and any change was assessed by Student's $t$ test for paired data.

The diary card mean scores for six of the patients with persistently purulent secretions were analysed separately. These patients had initially received low dose amoxycillin and were categorised by SLH as having failed to respond. After treatment with the higher oral dosage regimen, however, they were categorised as having responded. Their cards from the two treatment courses were compared. This enabled a clearer assessment of the patients' interpretation of their sputum characteristics and symptoms to be obtained since they acted as their own controls.

The difference between two time points for each symptom for both the successful and the unsuccessful treatment regimens was compared statistically with Student's $t$ test for paired data.

\section{Results}

\section{RESPONSE TO ANTIBIOTICS}

Thirty three patients initially received low dose amoxycillin and macroscopic clearance of sputum was achieved in $17(51.5 \%)$. All seven patients who were treated for a clinical exacerbation when sputum became purulent or mucopurulent (the patients who usually produced mucoid sputum) responded to this regimen and had a prolonged remission (median $61 / 2$, range 1-11 months). Similarly, the seven patients who regularly produced secretions fluctuating from mucopurulent to purulent had a macroscopic response, though six relapsed within 14 days and the remaining patient at 31 days (median nine, range 4-31 days). Of the 19 patients producing persistently purulent sputum, however, only three responded and they relapsed at, respectively, 4,4 , and 5 days.

Twelve of the 16 patients who failed to respond to low dose amoxycillin subsequently received high dose amoxycillin. Three patients withdrew because of reluctance to cooperate further with the study and one was withdrawn because of the development of a rash attributable to penicillin. All were patients who usually produced persistently purulent secretions. A macroscopic sputum response was observed in seven of these 12 patients $(58.3 \%)$ and their sputum remained clear for longer than the three such patients whose secretions had cleared with low dose amoxycillin (median 14, range 5-24 days). In view of the small numbers statistical analysis was not possible.

Three of the five remaining patients received nebulised amoxycillin and two showed a clear response during four months of treatment, their sputum having remained clear for six and 11 months so far after cessation of treatment. The other two patients died from unrelated causes before progressing to this regimen.

Cumulative analysis of results from the 27 patients who completed the study (exclusions not associated with failure of the regimen) showed that clearance of sputum from purulent to mucoid can be achieved in $96 \%$ of them with the amoxycillin regimens described. In $60 \%$ the sputum cleared with low dose amoxycillin, but this proportion rose to $90 \%$ when the high dose amoxycillin responders were included.

\section{BACTERIOLOGY}

Several organisms were isolated by the bacterial cultures before antibiotic treatment in the 17 patients who responsed macroscopically to low dose amoxycillin. These included eight organisms sensitive to amoxycillin (6 Haemophilus influenzae, 2 Streptococcus pneumoniae) and a penicillin resistant mucoid strain of Pseudomonas aeruginosa. No clear pathogen was identified in the remaining eight.

Of the 16 patients who failed to respond to this initial treatment regimen, a sensitive $H$ influenzae strain was isolated in five. Resistant $H$ influenzae was isolated in three patients, resistant Staphylococcus aureus in one patient, non-mucoid Pseudomonas aeruginosa in two, and no pathogen in five.

The five patients from whom sensitive $H$ influenzae was grown, and who did not respond to low dose amoxycillin, showed a clear response to the higher dosage. A response was also seen in a further patient, from whom a resistant $H$ influenzae ( $\beta$ lactamase negative) had been isolated.

The two patients who subsequently responded to nebulised amoxycillin were producing sputum from which apparently resistant organisms were isolated (Staph aureus and Pseudomonas). A penicillin resistant $H$ influenzae was still isolated from the sputum from the patient who failed to respond to this treatment. 


\section{PATIENTS' DIARY CARD ASSESSMENTS}

Frequency analysis of the diary card symptom scores from all the patients categorised by SLH as having responded showed significant improvements in sputum volume $(p<0.02)$, sputum thickness $(p<0.05)$, general well being $(p<0.01)$, dyspnoea $(p<0.05)$, and cough $(p<0.02)$ from before to during antibiotic treatment. No significant differences were found for any of the diary card scores for the patients categorised as not having responded.

The results for sputum colour scores are represented as frequency histograms (as shown in fig 1) for the groups, identified by SLH as having responded and as having not responded at all three analysis points (immediately before, during, and after the two oral regimens). The patients noted significant improvements in scores if they had been identified by SLH as having responded to treatment $(\mathrm{p}<0.001)$. This improvement remained two weeks after treatment had ceased, although patients had indicated a partial return to pretreatment scores (fig l).

When those identified as responders were divided into the three subgroups according to the usual
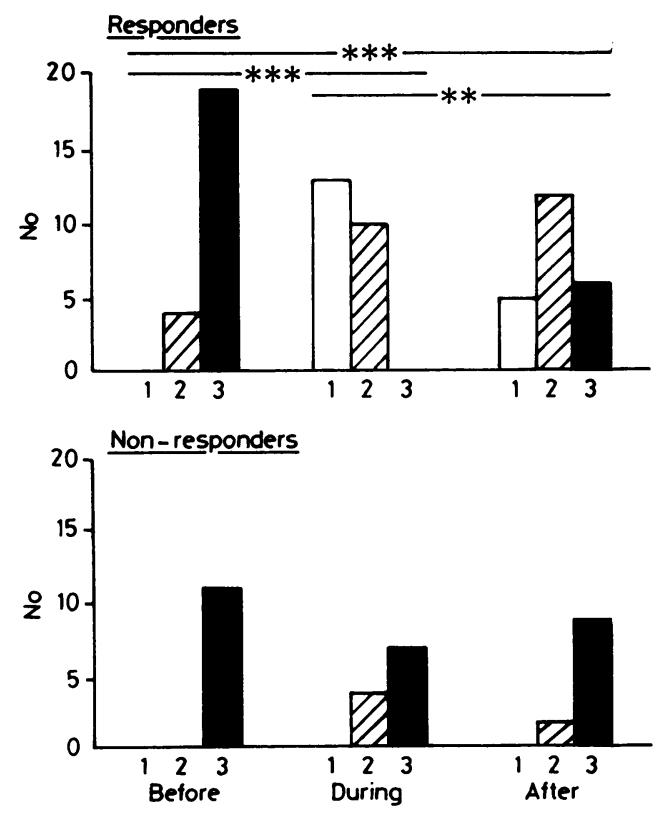

Fig 1 Frequency histograms for sputum colour scores (3-purulent; 2-mucopurulent; 1 -mucoid) derived from the patients' diary cards before, during, and after antibiotic treatment. The results are shown for the whole group of responders and non-responders (on the basis of the

macroscopic assessment of sputum by an observer ( $S L H)$ ). Significance levels are shown $\left({ }^{* * *} p<0.001 ;{ }^{* *} p<0.01\right.$; $\left.{ }^{*} p<0.05\right)$ for the comparison between treatment times (before to during, before to after, during to after) where statistical significance was attained. nature of their secretions when clinically stable (that $\stackrel{x}{*}$ is, $\mathrm{PP}, \mathrm{MP} / \mathrm{P}, \mathbf{M})$ there was no overall difference in $\overrightarrow{\overrightarrow{5}}$ most of the symptoms. The mucoid group alone showed an improvement in well being $(\mathrm{p}<0.05)$ and the persistently purulent group alone an improvement $\frac{\bar{\sigma}}{\bar{G}}$ in cough $(\mathrm{p}<0.05)$. All three groups, however, $\stackrel{\vec{\Phi}}{\vec{D}}$ showed a significant change in sputum colour scores with treatment (fig 2).

Patients from the PP and MP/P groups received $\overrightarrow{0}$ treatment in the apparently stable clinical state, unlike those in the $\mathbf{M}$ group, who were treated $\vec{\omega}$ because of a clinical exacerbation. The symptom scores for these two groups were therefore combined $x$ for further analysis. The results showed an $\rightarrow$ improvement in cough similar to that in the PP group $V$ alone. In addition, the combined group showed an improvement in well being $(\mathrm{p}<0.05)$, which disappeared two weeks after treatment.

Peak expiratory flow values taken from the diary $\overrightarrow{ }$ card showed a significant improvement $(\mathrm{p}<0.001)$ in the responding group as a whole (mean before 243.4 (109.6) $1 \mathrm{~min}^{-1}$; mean during $\left.267.3(114.1) 1 \mathrm{~min}^{-1}\right) . \vec{\emptyset}$ When subdivided into the three groups a significant improvement ( $\mathrm{p}<0.02$ and $<0.05$ respectively) was observed in both the PP group (mean before 195 (71.3) $1 \mathrm{~min}^{-1}$; mean during $\left.229(102.5) 1 \mathrm{~min}^{-1}\right)$ and the $\mathbf{M}$ group (mean before $222(94.0) 1 \mathrm{~min}^{-1}$; mean during 242 (89.7) $1 \mathrm{~min}^{-1}$ ) but no significant change $\stackrel{\mathbb{Q}}{\mathbb{Q}}$ was seen in the MP/P group (mean before $330 \stackrel{F}{F}$ (126.7) $1 \mathrm{~min}^{-1}$; mean during 343 (124.7) $\left.1 \mathrm{~min}^{-1}\right)$. No윽 differences were observed for PEF after a course of antibiotics that failed to produce a change in sputum colour.

Six patients were able to act as their own controls. All had received two oral courses of amoxycillin but $\underset{x}{\grave{2}}$ had shown a response (as assessed by SLH) only to $\overline{0}$ the higher dose regimen. When they failed to respond 3 . during the low dose treatment analysis of the diary 8 card showed no change in any symptom or in their 3 sputum characteristics. When they responded to the 0 higher dose regimen (assessment by SLH), however, the diary card scores for sputum colour $(\mathrm{p}<0.001)$ 을 and cough $(\mathrm{p}<0.025)$ improved during treatment.

\section{Discussion}

The present study was designed to investigate the $\omega$ response to antibiotics in patients with bronchiectasis who were under close supervision with the help of a patient diary card. In this respect a clear change of $\stackrel{\mathscr{S}}{-}$ expectorated secretions from purulent to mucoid or 7 slightly mucopurulent was assessed by one of us $\bar{O}$ (SLH) and compared with the patient's subjective assessment. During the initial observation period it $\frac{}{\mathbb{D}}$ was observed that expectoration from these patients fell into three categories, as described under 

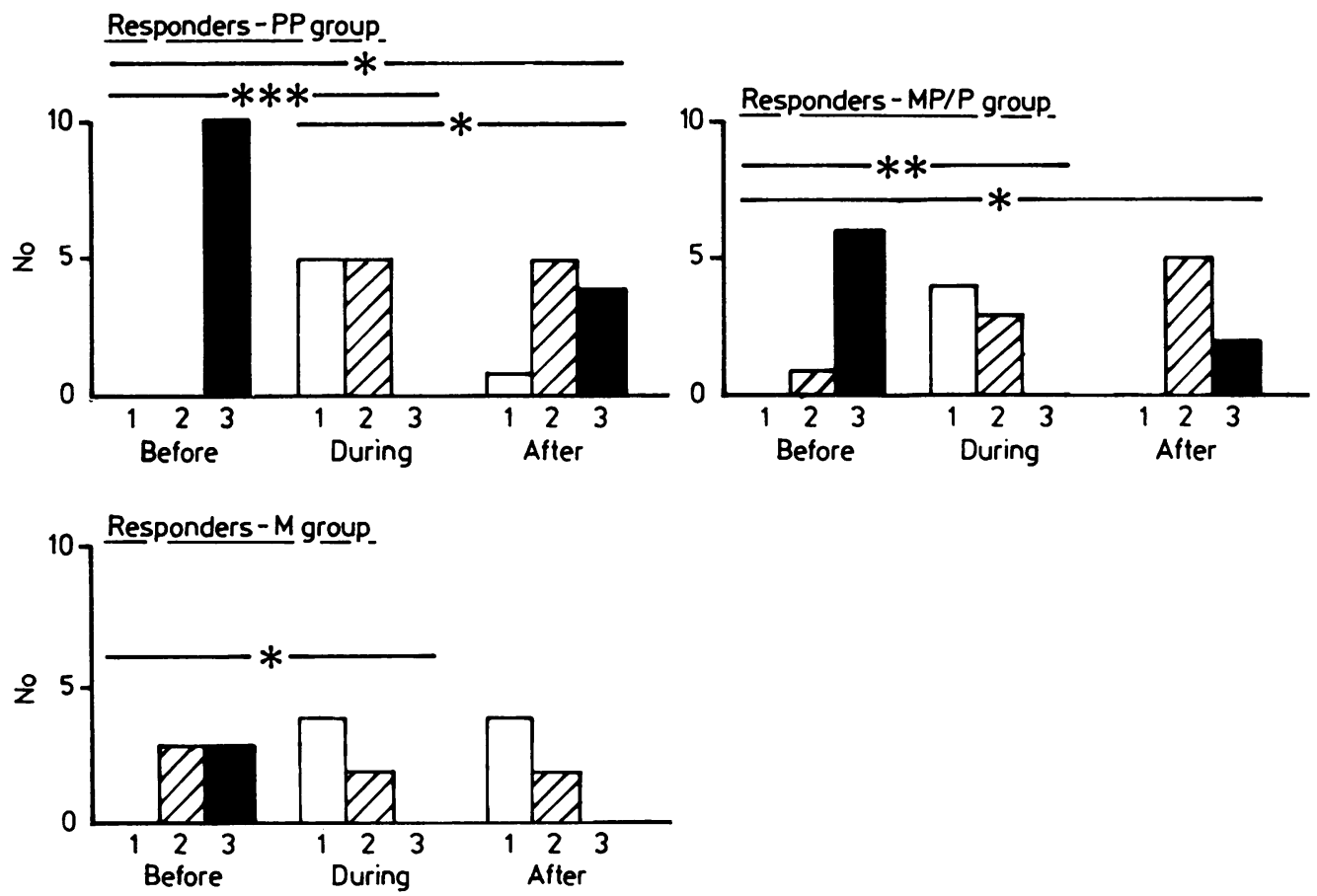

Fig 2 Frequency histograms for sputum colour scores (see fig 1) for whole group of responders divided into the three groups of patients identified $(M, M P / P, P P)$. Significance levels are shown $\left({ }^{* * *} p<0.001 ;{ }^{* *} p<0.01\right.$; $\left.{ }^{*} p<0.05\right)$ for the comparison between treatment times (before to during, before to after, during to after) where statistical significance was attained.

"Methods." Patients were then studied during and after antibiotic treatment. In the case of the mucoid group of patients this was during a clear clinical exacerbation with purulent sputum, when antibiotics would be conventionally prescribed. The mucopurulent-purulent and persistently purulent groups were, however, treated during the apparently stable clinical state. The reason for treating these groups was based on our previous data, ${ }^{2}$ which showed that purulent secretions were associated with detectable elastase activity, an increased inflammatory response in the lung, and worse lung function. We were thus interested in whether treating such patients elicited a response that was accompanied by an improvement in well being and peak expiratory flow.

Amoxycillin was capable of clearing the purulent secretions in most patients $(96.7 \%)$, although the dosage appeared to be important. Sputum from patients in the $\mathrm{M}$ and MP/P groups cleared macroscopically with low dose treatment. In patients producing persistently purulent secretions, however, this regimen rarely cleared the sputum. Clearance was seen in these patients with higher dosages $(60 \%)$, although some required nebulised drug before it was achieved.
The failure of broad spectrum antibiotics to clear purulent secretions in these patients has been noted by us previously. ${ }^{5}$ There are several possible reasons for failure. Firstly, it could be due to the presence of resistant organisms. Although some such organisms were isolated, their presence did not predict the response, confirming our previous findings. ${ }^{5}$ Furthermore, the same antibiotic cleared the secretions of most patients, provided that a sufficient dosage was used. This suggests that isolation of a particular pathogen and identification of its in vitro antibiotic sensitivity may not be important in these patients. Perhaps an overall reduction of the colonising microbial load may be more relevant than the removal of a single pathogen in these patients, as suggested by Cole and his colleagues. ${ }^{8}$

Secondly, failure of antibiotic response may be associated with inadequate penetration of the antibiotic into the bronchial secretions. It is well known that amoxycillin penetrates both purulent and mucoid sputum effectively, ${ }^{9}$ although Cole et $a l^{6}$ have suggested that increased doses are required in some patients. This is confirmed by the present study. Patients who produced persistently purulent sputum usually required the higher dose and some even 
required the drug to be delivered directly into the airway. This seems unlikely to be due to failure of antibiotic penetration, since amoxycillin penetrates tissues well and the lungs of these patients showed evidence of inflammation, ${ }^{2}$ which would further assist penetration owing to increased transudation from blood.

To be effective, however, the antibiotic level must exceed the minimum inhibitory concentration for the organisms causing infection. Cole has shown that such levels measured by bioassay are achieved only with a higher dosage of amoxycillin. ${ }^{6}$ This may represent true failure of antibiotic penetration, although inactivation by $\beta$ lactamase production by the bacteria is a possibility. In the current study $\beta$ lactamase producing organisms were rarely isolated. It does, however, remain possible that $\beta$ lactamase is present in these secretions as a result of production by organisms such as anaerobes that were not readily cultured. This might explain why larger doses are required to achieve the minimum inhibitory concentration as measured by bioassay: the larger doses of antibiotic in these patients might overcome in situ inactivation and lead to a therapeutic response. Clearly, further studies are required to clarify these possibilities. The additional response of some patients when a lower dose of the drug was given by the nebulised route suggests, however, that factors governing local antibiotic concentration are the major determinants of response in these patients.

The patients' diary cards illustrated how well they were able to assess their own sputum colour and these records mirrored our own observations (figs 1 and 2). Those whom we categorised as responders and as non-responders on the basis of the macroscopic assessment by SLH detected similar changes with their cards. In addition, the patients observed an improvement in symptoms only when they were categorised by us as having responded (see under "Results"). Analysis of the individual groups (M, MP/P, and PP) failed to show significant changes in most of the symptoms, probably owing to small numbers.

The improvement in well being seen in the $\mathbf{M}$ group was expected since they were treated when clinically "unwell." The MP/P and PP groups together, however, who were treated in the stable clinical state, also noted an improvement in well being. This could, of course, reflect patients' bias in the assessment of treatment. No such improvement, however, was noted in the group classified as not having responded to an antibiotic. Furthermore, a real change is supported by the six patients with persistently purulent sputum who acted as their own controls. No change was noted by these patients when the sputum failed to show a response, whereas the results were similar to those for the larger group when they did respond. The overall improvement in well being in this group of patients suggests that they are not usually completely $\frac{\overrightarrow{0}}{0}$ well, despite conventional belief. Furthermore, recent $\frac{}{5}$ evidence suggests that their serum acute phase protein $\overline{\bar{m}}$ concentrations are raised, ${ }^{10}$ providing additional $\overparen{\nabla}$ evidence that active treatment may be appropriate in them.

The clinical course of patients after response to $\overrightarrow{0}$ antibiotic was variable. The $\mathbf{M}$ group had long $\overrightarrow{\vec{C}}$ periods before their sputum became purulent again $\omega_{\sigma}$ but the patients in both the MP/P and the PP groups $\overrightarrow{\overrightarrow{2}}$ relapsed rapidly. This may be important, since puru- $x$ lent secretions tend to be associated with elastase activity and have deleterious effects on the ciliated iv airway, ${ }^{311}$ and, as we have suggested, these patients of may not be entirely well. Clearly, if these factors are 0 important in both the pathogenesis and the pro- $工$ gression of the disease such short courses of anti- $\vec{c}$ biotics would be inappropriate. The patients who received nebulised drug were treated for longer (four $\vec{A}$ months) and the two who responded had longer remissions before the sputum again became purulent. The significance of this requires further study, however, to determine the success of longer courses of treatment with all three regimens.

Small but significant changes (about 10\%) were observed in the peak expiratory flow after response to $\stackrel{\mathbb{Q}}{\varrho}$ antibiotic. The reasons for this change are uncertain $\overrightarrow{\vec{O}}$ since most of the patients did not show reversibility of $\frac{3}{3}$ airflow obstruction with formal bronchodilator chal- $\bar{P}$ lenge. It remains possible that the failure of formal bronchodilator testing is due to mucus and oedema of the airways related to the presence of purulent secretions and inflammation.

Further studies of lung function in these patients $\dot{\sigma}$ failed to show any major acute changes even after 3 . four months of antibiotic treatment (unpublished 0 observations). This is at variance with the acute changes found by Cole $e t a l^{6}$ after only seven days of $\bigcirc$ antibiotic treatment and recently reported in a British $\rightarrow$ Medical Journal leading article, ${ }^{12}$ suggesting that the patients we studied differ from those studied by Cole and colleagues.

In conclusion, amoxycillin clears the purulent $\tilde{O}$ secretions of patients with bronchiectasis, although the dose is critical. Few patients with persistently purulent secretions respond to conventional lowe dosage, whereas those who usually produce mucoid $\frac{}{\Phi}$ sputum usually respond to conventional doses. The $\stackrel{\infty}{+}$ macroscopic responses observed are associated with $\square$ subjective improvement in the patients' symptoms but with only small changes in lung function. Relapse is rapid in patients in the MP/P and PP groups, sug- $\overrightarrow{\mathbb{Q}}$ gesting that longer courses of antibiotic treatment $\bar{O}$ may be indicated. 
We acknowledge the financial support of the General Hospital bicentenary appeal fund, the Edgar Lawley Trust, and Bencard. We thank Miss K Roberts and Mrs EP Ford for typing the manuscript and $\mathrm{Mr} \mathbf{R}$ Holder (department of statistics, University of Birmingham) for valuable advice on the statistical analysis of the data. RAS is a Wolfson research fellow of the Royal College of Physicians of London.

\section{References}

1 Medical Research Council. Prolonged antibiotic treatment of severe bronchiectasis. Br Med J 1957;II:255-9.

2 Stockley RA, Hill SL, Morrison HM, Starkie CM. Elastolytic activity of sputum and its relation to purulence and to lung function in patients with bronchiectasis. Thorax 1984;39:408-13.

3 Smallman LA, Hill SL, Stockley RA. Reduction of ciliary beat frequency in vitro by sputum from patients with bronchiectasis: a serine proteinase effect. Thorax 1984;39:663-7.

4 Lourenco RV, Loddenkemper R, Carton RW. Patterns of distribution and clearance of aerosols in patients with bronchiectasis. Am Rev Respir Dis 1972;106:857-66.
5 Stockley RA, Hill SL, Morrison HM. Effect of antibiotic treatment on sputum elastase in bronchiectatic outpatients in a stable clinical state. Thorax 1984;39:414-9.

6 Cole PJ, Roberts DE, Davies SF, Knight RK. A simple oral antimicrobial regimen effective in severe chronic bronchial suppuration associated with culturable Haemophilus influenzae. J Antimicrob Chemother 1983;11: 109-13.

7 Stockley RA, Hill SL, Burnett D. Nebulised antibiotics in chronic purulent bronchiectasis. Clinical Therapeutics 1985;7:593-9.

8 Cole PJ, Roberts DE, Higgs E, Prior C. "Colonising microbial load": a cardinal concept in the pathogenesis and treatment of progressive bronchiectasis due to "vicious circle" host mediated damage [abstract]. Thorax 1985;40:227.

9 May JR. The chemotherapy of chronic bronchitis and allied disorders. 2nd ed. London: English Universities Press, 1972:46-8.

10 Stockley RA. Chronic bronchial sepsis. $\mathrm{Br}$ Med J 1985;290:1430.

11 Tegner H, Ohlsson K, Toremalm NG, Von Mecklenberg C. Effect of human leukocyte enzymes on tracheal mucosa and its mucociliary activity. Rhinology 1979;17:199-206.

12 Cochrane GM. Chronic bronchial sepsis and progressive lung damage. $\mathrm{Br}$ Med J 1985;290:1026-7. 